coefficient as low as $10^{-22} \mathrm{~cm}^{2}$ see. $^{-1}$ would be required, instead of the actual value of about $10^{-5} \mathrm{em} .^{2}$ sec. ${ }^{-1}$. The diffusion to the surface is therefore followed by a process of high activation energy to produce the final state of the surface film. In eonfirmation of this, the rate at which the final interfacial tension is reached has been found to increase sharply with rising temperature.

The presence of electrolytes in the aqueous phase has been found to alter the interfacial tension - time variation. With this system, where the electrolyte is in a different phase from the surface-active solute, it can exert a direct influence on the interface without altering the diffusion-rate, degree of aggregation, ete., of the solute in the oil phase.

By expanding and eontracting the pendent drop, the closeness of packing of the molecules adsorbed at the interface could be varied and $F-A$ eurves obtained. The interfacial films behaved like insoluble surface films, since a compression of the film did not drive molecules back into solution. Therefore the activation barrier affects desorption as well as adsorption of molecules.

College of Technology,

A. F. H. WARD.

L. Tordai.

Manchester.

June 22.

${ }^{1}$ Doss, K. S. G., Koll. Z., 84, 138 (1938) and 86, 205 (1939).

${ }^{2}$ Adam, N. K., and Shute, H. L., Trans. Farad. Soc., 34, 758 (1938). 'Alexander, A. E., Trans. Farad. Soc., 37, 15 (1941).

- Ward, A. F. H., and Tordai, L., J. Sci. Instrum., to appear shortly.

${ }^{5}$ Andreas, J. M., Hauser, E. A., and Tucker, W. B., J. Phys. Chem., 42, 1001 (1938).

\section{Light-Effect in Chlorine under Electrical Discharge: Change of the Wave Form due to Irradiation}

Previous work on this phenomenon ${ }^{1,2,3}$ showed that a current decrease $\Delta i$ is produced by irradiation of chlorine and other gases referred to the rectified mean r.m.s. values of $i$ under different conditions; changes in the corresponding current-structure and its time-delineation are revealed by a cathode ray oscillograph $^{3}$. An iron core step-down transformer was introduced in series with an A.c. indicator. Its secondaries were connected to one pair of the deflecting plates in the oscillograph; the other pair was connected to the time-sweep potential. With but a moderate amplifier gain on each of the plate pairs and the usual adjustments, the oscillograph revealed, besides the frequeney of the A.c. supply, a remarkably large number of higher frequencies of widely varying strengths; they were not transients but repeatable and charaicteristic eonstituents of $i$ the discharge current. It was also striking to observe the sensibly instantaneous and reversible diminution of the amplitudes of these component frequencies, on irradiating the dischargè tube, as shown by a typical pair of oscillograms reproduced herewith.

It was observed ${ }^{1,4}$ that the production of a change under electrical discharge, chemical or otherwise, for example, the hydrogen chlorine reaction, the activation of nitrogen, its deactivation, etc., requires a minimum threshold potential $V_{m}$ characteristic of the reaction, its operative conditions such as the frequeney $n$, the temperature, etc. At $V_{m}$, the wattage dissipated in the system and $i$ show a rapid rise

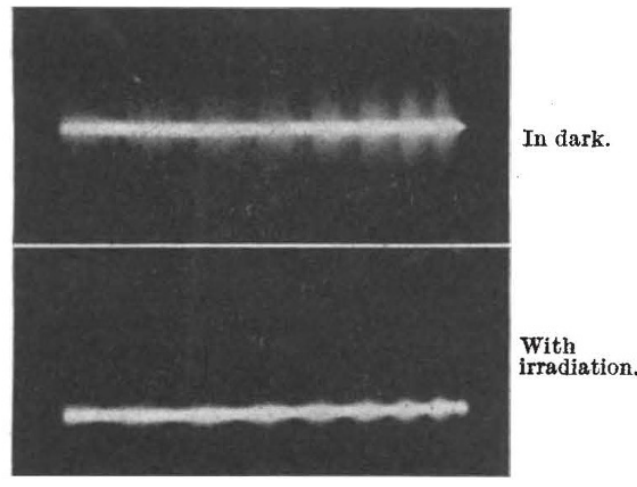

Exposure, $2 \mathrm{~min}$.

as $V$ is increased. In elementary gases, $V_{m}$ may be identified with or related simply to the corresponding Paschen potential. Below $V_{m}$, the oscillograph showed a markedly simple current-structure ; further, changes of the amplitudes due to the light-effect were not detected. It is considered that ionization by collision in fields due to the applied potential is a necessary condition for the occurrence of this phenomenon. It is found that both for the light-effect and the above type of reactions, $V_{m}$ diminishes as $n$ is increased; its influence on the light-effect expressed as $\Delta i / i$ is also in the same direction.

An examination of a number of oscillogram pairs like those reproduced, obtained under different conditions, showed that the proportionate reduction on irradiation of the component amplitudes was fairly uniform. These higher frequencies in $i$ represented both the audio and radio ranges. When the latter were eliminated with a series of H.F. filters, the light-effect in the residual audio range was similar to that for the total or unfiltered current. That the above remark applies also to the radio range was indicated by similar results for the light-effect $\Delta i / i$ observed, with and without an amplifier, for $i$ picked up by a moderate size aerial 1-9 ft. distant from the chlorine tube.

Chemical Laboratories,

S. S. Joshi.

Benares Hindu University. May 8.

2 Joshi, Curr. Sci., 8, 548 (1939).

'Joshi, Pres. Address, Chem. Sec., Ind. Sci. Cong. (1943).

${ }^{8} \mathrm{Joshi}$, Benares. Hindu Univ. J., 8, 99 (1943).

- Joshi, Trans. Farad. Soc., 25, 120 (1929).

\section{Lanosterol}

THE problem of the origin and chemical nature of lanosterol-the eharacteristic substance so far observed only in the wool-grease of the sheep-was originally solved by classifying it as a sterol. Recent evidence $\Theta^{1,2}$ indicates a more probable connexion with the tri-cyclic terpenes such as $\alpha$-elemolic acid and with the mínor yeast sterol cryptosterol.

Among the oxidation products of cryptosterol are a number of ketonic derivatives which are yellow in colour, probably due to the presence. of a chromo-

phore grouping such as $-\mathrm{CO}-\mathrm{C}=\mathrm{C}-\mathrm{CO}-$. Ruzicka $^{3}$ has lately obtained similar yellow compounds, for example, aeetyl-iso-elemendionol acid ester, from the 